\title{
A Preliminary Study on Cathodic Prevention in Reinforced Mortar
}

\author{
D.A. Koleva ${ }^{\mathrm{a}}$, K.van Breugel ${ }^{\mathrm{a}}$, J.M.C. Mol ${ }^{\mathrm{b}}$, J.H.W. de Wit ${ }^{\mathrm{b}}$ \\ ${ }^{\mathrm{a}, \mathrm{b}}$ Delft University of Technology, The Netherlands \\ ${ }^{\mathrm{a}}$ Faculty of Civil Engineering \& Geosciences, Dep. Mater.\& Environment, \\ Stevinweg 1, 2628 CN Delft \\ ${ }^{\mathrm{b}}$ Faculty 3mE, Dep. Mater. Sci.\&Eng., Mekelweg 2, 2628 CD Delft \\ This work presents the preliminary tests on the performance of \\ cathodic prevention (CPre) in reinforced mortar, subjected to \\ aggressive (10\% $\mathrm{NaCl}$ environment). Cathodic prevention is an \\ electrochemical technique for minimizing, actually "preventing" \\ any eventual corrosion of the steel bars in reinforced concrete. \\ Therefore, the main objective of this investigation is to monitor the \\ performance of cathodic prevention (CPre) as an alternative of \\ cathodic protection (CP) i.e. application before corrosion initiation, \\ using low cathodic current densities (1 to $2.5 \mathrm{~mA} / \mathrm{m}^{2}$ steel surface). \\ The novelty here is the application of "pulse" cathodic prevention \\ (pulse DC current), rather than the conventional steady DC \\ technique. The paper reports on conventional monitoring (potential \\ mapping and depolarization decay), coupled with visualization of \\ the steel/cement paste interface, using SEM and EDX.
}

\section{INTRODUCTION}

Well known is that chloride-induced localized corrosion of the embedded steel is one of the most hazardous types of corrosion in reinforced concrete $(1,2)$. Cathodic protection (CP) is one of the electrochemical techniques, used for corrosion protection in reinforced concrete (3-10). The widespread use of CP is well established, some developments in the field have been reported elsewhere $(5,7)$. The application of CP however is accompanied by negative side effects (e.g. alkali-silica reaction, bond strength degradation, etc.), some of them reviewed in $(3,7)$.

Cathodic prevention (CPre) is a useful technique, which would prevent steel corrosion in reinforced concrete, if applied before corrosion initiation. Since CPre uses significantly lower current density, compared to the conventional CP (1 to $3 \mathrm{~mA} / \mathrm{m}^{2}$ for the former versus $10-20 \mathrm{~mA} / \mathrm{m}^{2}$ for the latter), a significant reduction of negative side effects (as above mentioned) as well as maintenance costs in general, is expected with such applications.

Additionally, both CP and CPre can be applied using the conventional way of steady DC cathodic polarization, but also using a more novel approach, based on pulse technology. As far as CP is concerned, the so called pulse CP (using a square pulsed DC signal instead of steady DC, at 12.5 to $50 \%$ duty cycle and frequency $1 \mathrm{kHz}$, the current being the controlling parameter (11)) was proven to have superior performance, compared to conventional CP $(12,13)$. 


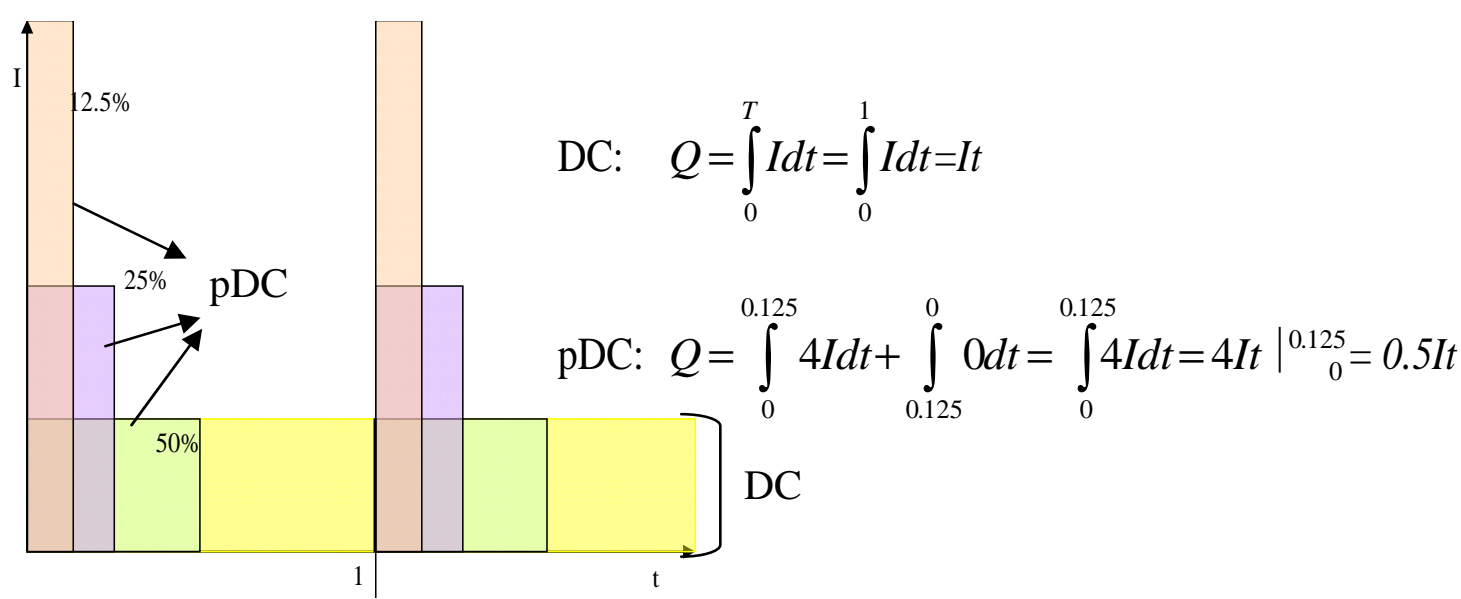

Fig.1 Schematic illustration of DC and pulse DC regimes and calculation (based on well known relationships, for pDC at duty cycle 12.5\%) of the electrical charge (and half of the applied within DC regime current density and 50\% charge, respectively) relevant to both DC the pulse DC.

To this end, the main objective of this preliminary study was to investigate the performance of pulse CPre (i.e. pulsed DC applied as prevention), compared to conventional CPre. The current density involved in CPre is at least 10 times lower, compared to CP ( 1 and $2.5 \mathrm{~mA} / \mathrm{m}^{2}$ hereby investigated). Furthermore, when pulsed CPre is used, the average current density involved is from 0.5 to $1.25 \mathrm{~mA} / \mathrm{m}^{2}$ since the pulse regime uses half of the conventionally applied cathodic current density, Fig.1, $(12,14)$. The performance of both conventional and pulse CPre was monitored via generally applied in practice techniques as potential mapping and depolarization decay. A more detailed presentation for the influence of the CPre techniques on electrochemical parameters of the steel reinforcement, coupled with microstructural analysis at the end of the test will be subject to a further contribution.

\section{MATERIALS AND METHODS}

The investigated specimens were reinforced mortar cylinders of $\mathrm{h}=150 \mathrm{~mm}, \mathrm{~d}=$ $40 \mathrm{~mm}$, with embedded "as received" construction steel FeB500 HWL of d=8mm (5 replicates per group and per condition). All specimens used OPC CEM I 42.5, water-tocement ratio 0.6, cement-to sand ratio 1:3. Curing of the specimens was performed for 7 days in fog room $\left(20^{\circ} \mathrm{C}, 98 \% \mathrm{RH}\right)$; conditioning was in a laboratory environment $\left(20^{\circ} \mathrm{C}\right.$, lab air) for the total duration of the test of 320 days. The environmental medium was a highly aggressive $10 \% \mathrm{NaCl}$ solution (specimens were immersed $1 / 3^{\text {rd }}$ of height for the total duration of the test). In addition to the ordinary mixture (as specified above), a modified with polymeric nano-aggregates mixture (in the form of $\mathrm{PEO}_{113}-\mathrm{b}-\mathrm{PS}_{218}$ coreshell micelles, concentration of 0.006 wt. \% per mortar weight) was also investigated. The modified mixtures are not particularly subject to discussion in this contribution, more details for these specimens are reported in (15). The reason for investigating these mixtures is only comparative, in terms of achieved polarization within the CPre and eventual influence on bulk matrix resistivity with regard to CPre. Thus sample designation is: OPC for the ordinary mixtures and PoOPC for the modified mixtures. The regimes of protections are denoted as pCP (pulse prevention) and CP (steady DC prevention). The experimental methods involved were as follows: OCP monitoring for all 
cells; Depolarization (Decay) measurements; SEM imaging of the steel/cement paste interface for all conditions (using ESEM Philips XL30); Chemical analysis for total chloride in the vicinity of the steel reinforcement was performed as well, using Volhard titration method.

\section{RESULTS AND DISCUSSION}

The cathodic polarization started immediately after immersion of the cells in the $10 \%$ $\mathrm{NaCl}$ solution. The cathodic current density was $1 \mathrm{~mA}$ per square meter of steel surface and increased to $2.5 \mathrm{~mA} / \mathrm{m}^{2}$ after 90 days. The pulse regime used a block (square) pulse at $1 \mathrm{kHz}$, duty cycle of $12.5 \%$ (Fig. 1) and a high impedance current source. Thus, the pulse regime uses $50 \%$ of the average current, used within steady CPre i.e. 0.5 until 90 days and $1.25 \mathrm{~mA} / \mathrm{m}^{2}$ later on.

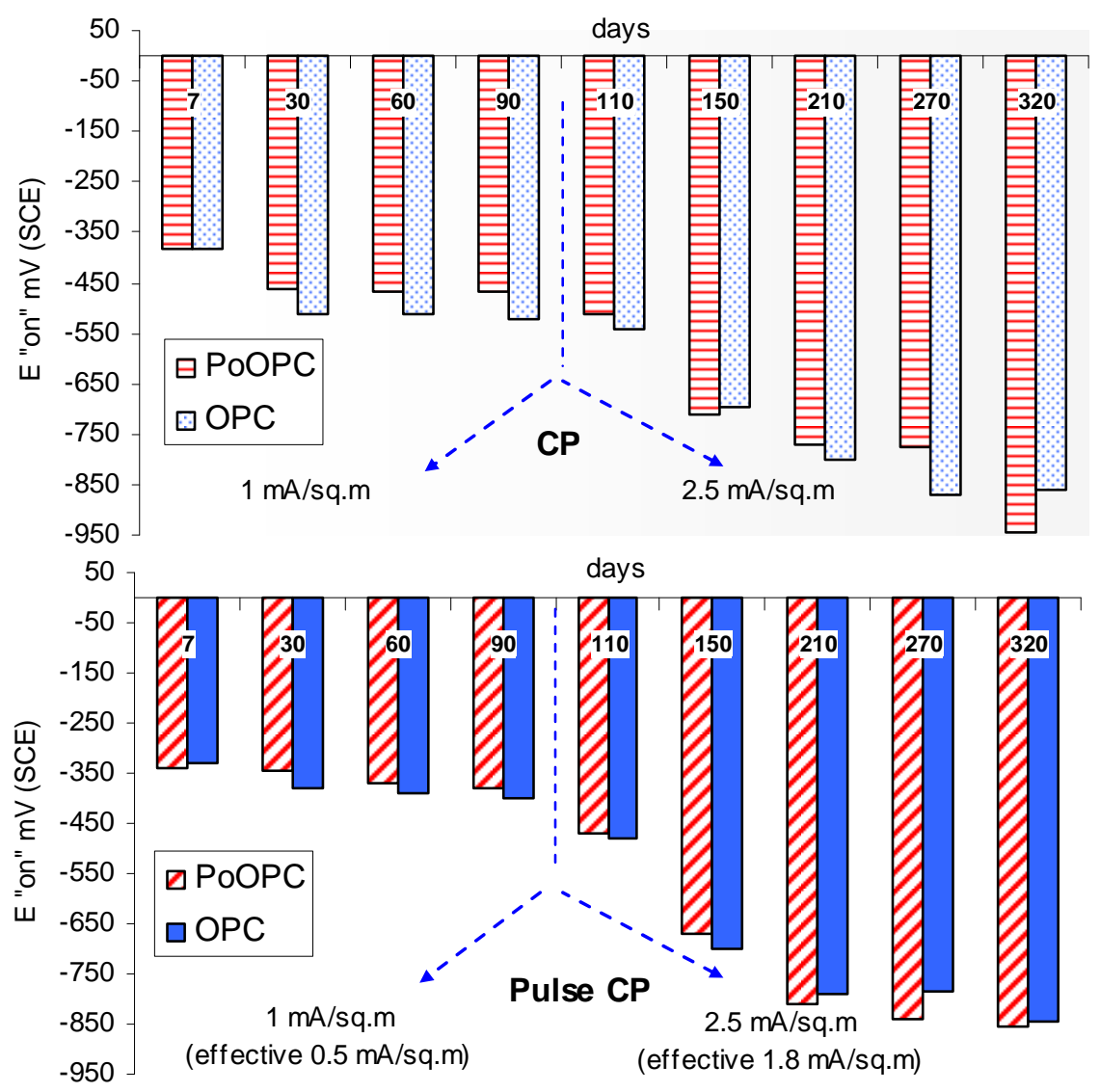

Fig.2 "ON" potentials for cells under conventional cathodic prevention (CP) - (a) and pulse cathodic prevention (pulse CP) (b) (PoOPC are cells, where the mortar mixture was modified with core-shell micelles, OPC are cells, using ordinary mortar mixture).

Figure 2 depicts the recorded "on" potentials for both regimes of prevention, showing also the comparison between samples with (PoOPC) and without (OPC) admixed micelles. As seen from the plots, similar polarization behavior was observed, with potentials reaching $-530 \mathrm{mV}$ (SCE) for the steady current CPre (Fig.2a) and approximately $-450 \mathrm{mV}$ (SCE) for the pulse CPre (Fig.2b). The current was increased after 90 days (as indicated in the plots) and cathodic polarization increased within the 
time interval of 320 days, reaching more than $-800 \mathrm{mV}$ in cathodic direction for both regimes.
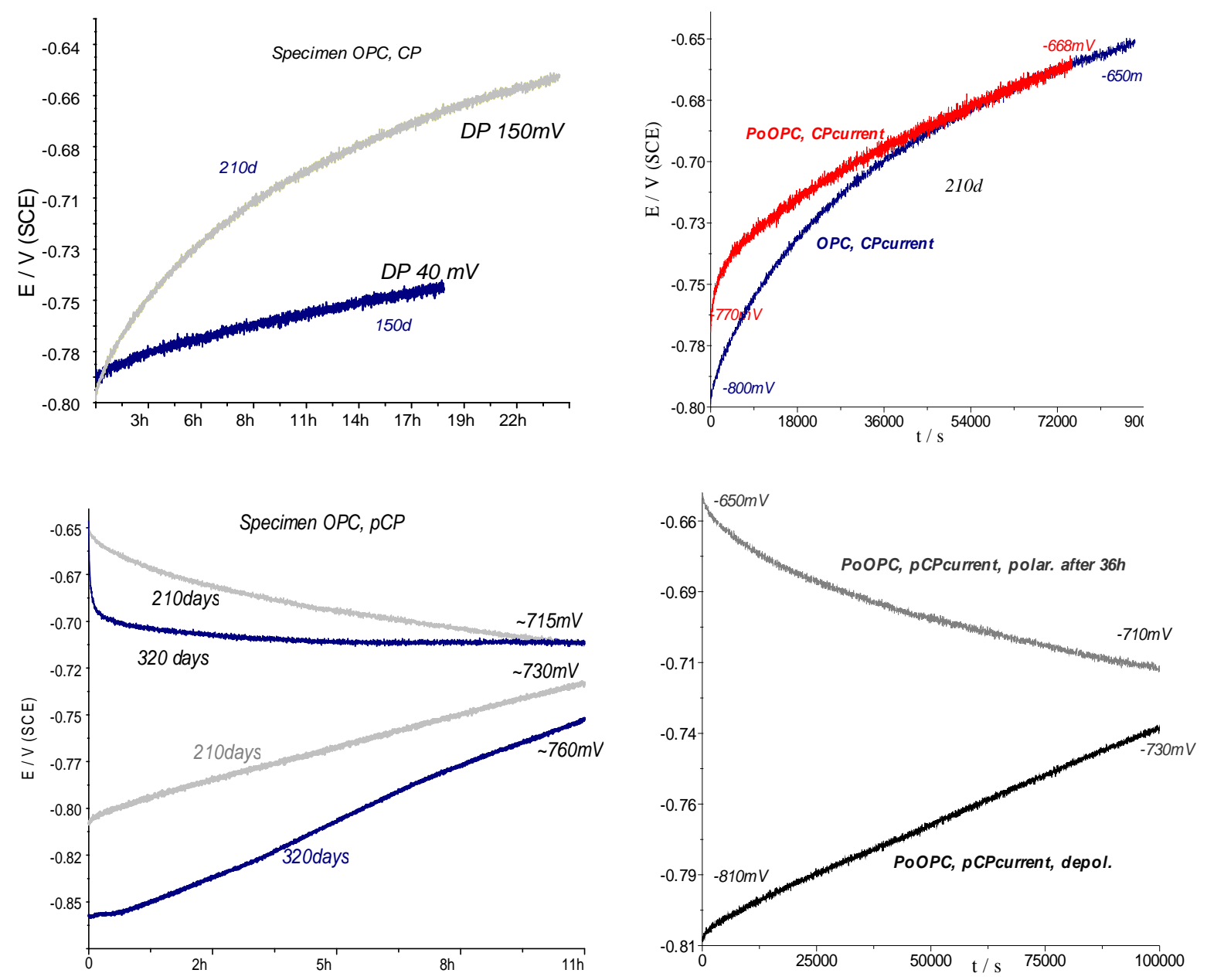

Fig.3 Decay measurements for: (a) specimens OPC under CPre (150 and 210 days); (b) a comparison of decay for specimens OPC and PoOPC under CPre, 210 days; (c) decay and polarization after decay for specimens OPC under pulse CPre and (d) decay and polarization for specimens PoOPC under pulse CPre.

Some considerations with respect to "ON” potential readings and decay measurements are necessary to be briefly pointed out. The conventional basis of Cathodic protection is polarizing the steel in the negative (cathodic) direction (16), which is maintaining the steel potential more cathodic than $-800 \mathrm{mV}$ (SCE) (according to standards, BSEN12696) where corrosion is thermodynamically impossible to occur (17). Thus the primary effect is reducing the driving force for the anodic reaction (thermodynamic effect) and retarding the anodic process (kinetic effect). The application of CP causes current flow, which in case of already initiated corrosion on the steel surface, will concentrate at the areas with lowest resistance (the anodic areas), so initially there will be a polarization of the anodic dissolution and decrease in corrosion rate (18). Further, two "protection" processes are expected: a classical thermodynamic immunity (long recognized $(17,19)$ ) or "imperfect passivity", which is a result from restored alkalinity of the environment at the steel/cement paste interface. In that sense, when CP is applied to reinforcing steel in concrete, the objective might not always be to achieve "immunity" i.e. cathodic 
polarization around $-800 /-850 \mathrm{mV}$ (SCE), but to reduce the risk of chloride-induced corrosion. This is the domain where CPre actually works, where a modest polarization of the steel surface will be sufficient for corrosion protection. With this respect, the so called "Pedeferri" diagram (6) gives visual correlation of the steel polarization, the involved phenomena and chloride concentration.

Summarizing, lower polarization (more anodic than $-850 \mathrm{mV}$ SCE potential readings) are sufficient within CPre applications, providing that chloride concentrations are relatively low and most importantly, the decay measurements show sufficient depolarization values. For the hereby investigated regimes, and as evident from Fig.2, the initial CPre, both conventional and pulse, result in potential values of about -350 to -500 $\mathrm{mV}$ (SCE) for 7 until 30 days of immersion. These fall in the so called "imperfect passivity zone" where pitting can not initiate, although can propagate in the case where corrosion has been previously initiated. The latter case is not relevant for the monitored cells, since CPre was applied immediately with immersion in the corrosion medium (for this type of system i.e. specimen geometry and mortar mixture, and for $10 \% \mathrm{NaCl}$ environment, corrosion initiates after 15 to 30 days after immersion due to chloride penetration and arrival at the steel surface (21)). Further, after 90 days of conditioning, the CP current was increased, and potential readings were more cathodic than $-800 \mathrm{mV}$ (SCE) for both pulse and steady CPre regimes.

Figure 3 depicts the Decay measurements, proving sufficient polarization i.e. the effective prevention in both pulse and steady CPre (according generally accepted criteria, e.g. NACE RP0290-2000, the decay should be approximately $70-100 \mathrm{mV}$ for $24 \mathrm{~h}$ ). Figure 3(a) presents a comparison of decay measurements for OPC cells under conventional (CP) prevention after 150 and 210 days of conditioning with decay values of $40 \mathrm{mV}(19 \mathrm{~h})$ and $150 \mathrm{mV}$ (22h). Figure 3(b) depicts an overlay of depolarization measurements for cells with and without admixed core-shell micelles at 210 days, again under conventional CPre, similarly showing sufficient decay of more than $100 \mathrm{mV}$ in $24 \mathrm{~h}$. Figures 2(c) and (d) depict depolarization and polarization curves for the pulse CPre regime (OPC specimens in Fig.3c and PoOPC specimens in Fig. 3d), again showing sufficient decay values and thus sufficient polarization i.e. effective performance of the pulse cathodic prevention technique.

Additionally, no corrosion products were detected on the steel surface after breaking open the specimens at 320 days (Fig.4c) d)), despite the significant chloride concentration of $\sim 5 \%$ in the bulk matrix (derived wet chemically). The lack of corrosion products in the specimens under CPre (both steady and pulsed) and the accumulation of such in nonprotected, corroding specimens, was also proved by SEM and EDX investigation of the steel/cement paste interface, performed at the end of the testing period (320 days). As a comparison of all conditions, Fig.4 presents the steel/cement paste interface in: a control (non-corroding, non-protected specimen), Fig.4a); in a corroding specimen, Fig.4b); and in protected specimens (both steady and pulse CPre) - Fig. 4c)d).

Further, a brief presentation of the on-going microstructural investigation is given in what follows, revealing the structural alterations due to electrical current flow. Fig. 5 presents the derived porosity and pore size distribution for specimens OPC, CPre and OPC, pCPre (in a comparison to reference (OPCref) and corroding (OPCcorr) specimens). At this stage of the investigation, the following observations are relevant: CPre current, 
both steady and pulse, bring about densification of the bulk matrix (as previously derived in different cement-based materials and at different hydration stages $(21,22)$ ). Additionally, chlorides are known to increase cement hydration i.e. bulk matrix densification (23-25), hereby evident by the lower porosity of specimens OPCcorr compared to OPCref and the lower critical pore size of the former, compared to the latter (Fig.5 right). The most relevant mechanism here seems to be a denser pore structure of the mortar cover and as a result, chlorides were not observed at the steel/cement paste interface in the protected specimens, evident from the EDX mapping results (Fig.4).

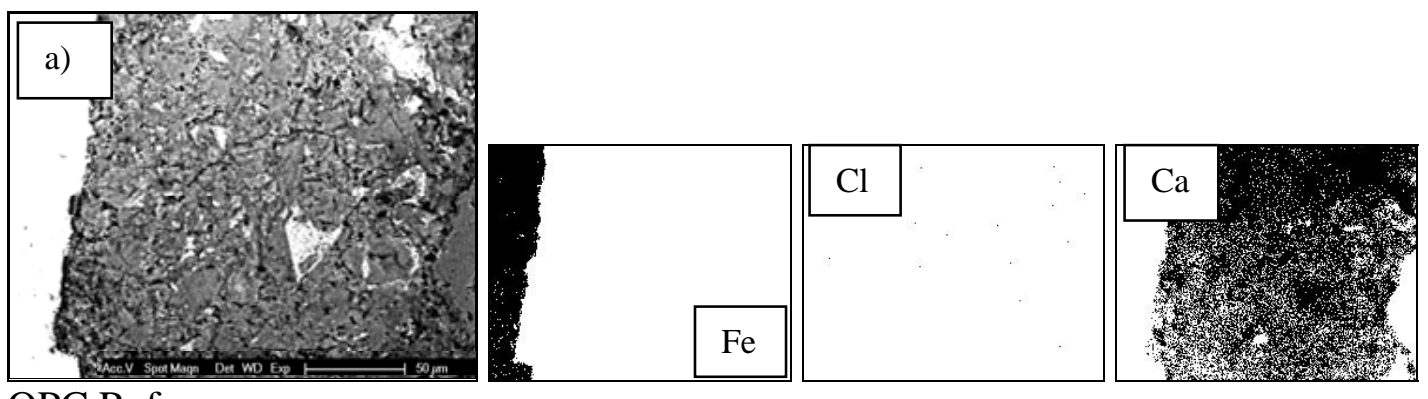

OPC Ref

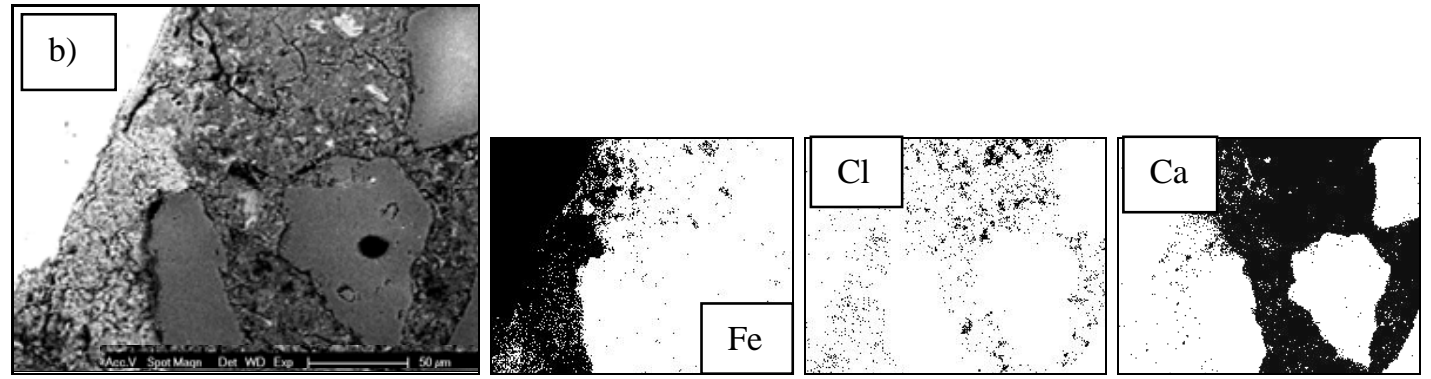

OPC corr
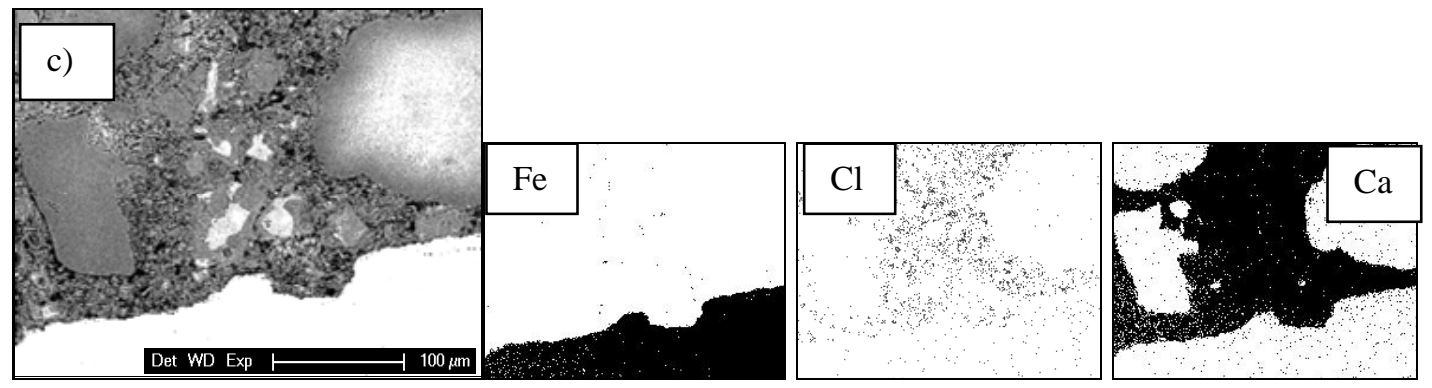

\section{OPC CPre}
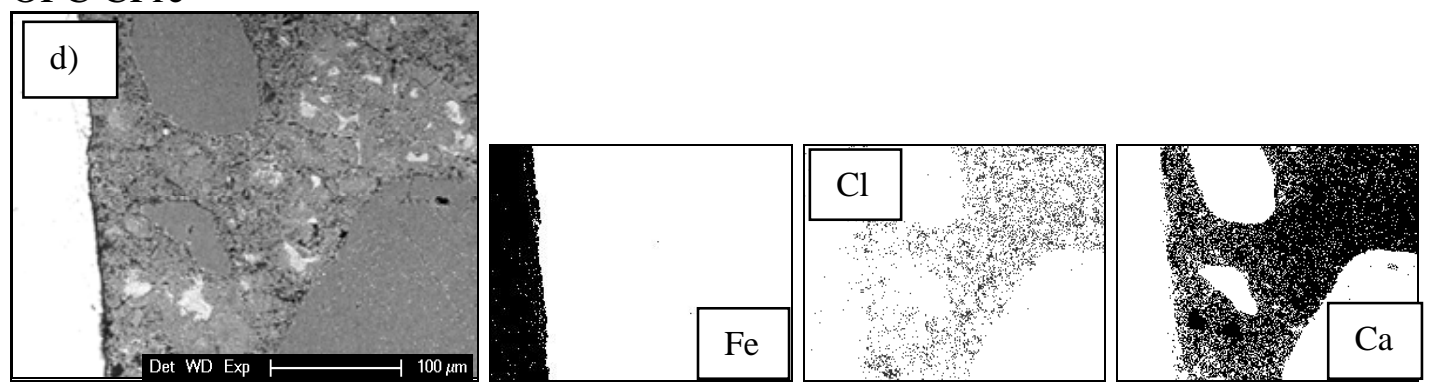

OPCpCPre

Fig.4 (a-d) SEM micrographs of the steel/cementy paste interface (left) and EDX elemental maps (right) 
For the specimens under CPre, a denser pore structure is relevant (Fig.5), which is a result from the combined effects of chloride binding and electrical current flow. Although chlorides were present at the steel/cement paste interface (Fig.4), as well as derived wet chemically (results not hereby discussed), corrosion was not initiated and observed respectively in these (CPre conditioned) specimens (Fig. 4). The relevant mechanism is a coupled effect of increased chloride binding and cathodic polarization of the steel surface due to CPre.
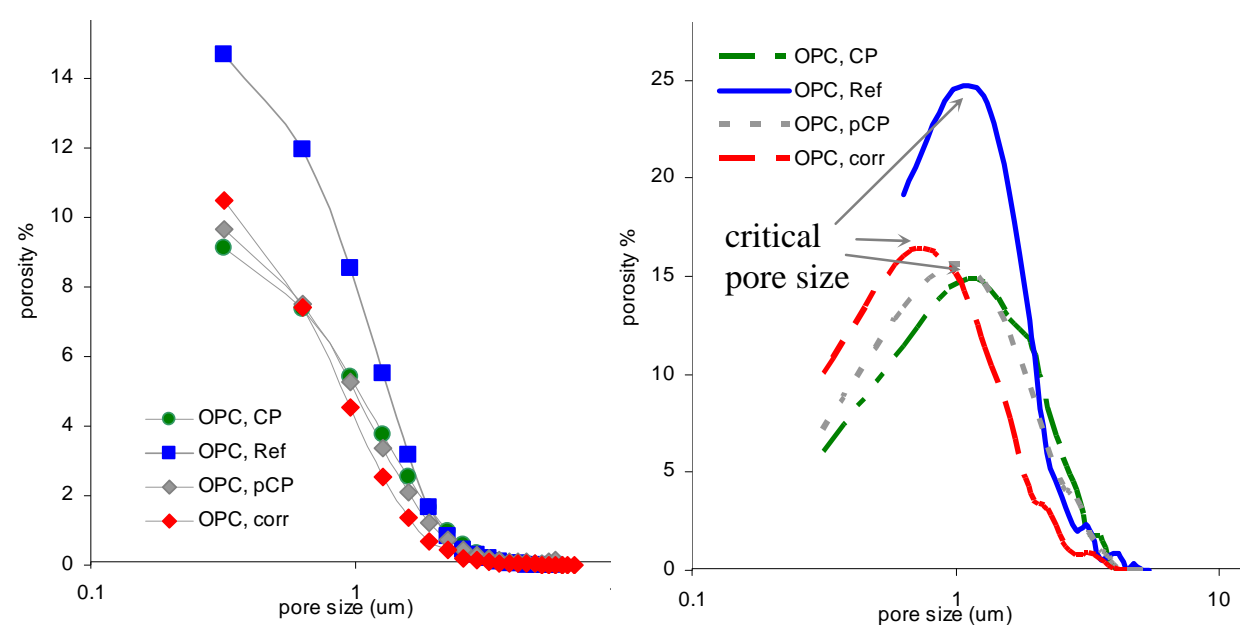

Fig.5 Bulk matrix porosity (left) and critical pore size (pore size distribution) (right) for the investigated specimens.

\section{CONCLUSIONS}

Both pulse and steady cathodic prevention, using very low current densities (in the range of 1 to $2.5 \mathrm{~mA} / \mathrm{m}^{2}$ ), are able to prevent corrosion initiation on the steel surface, even in an extremely aggressive environment such as $10 \% \mathrm{NaCl}$.

Cathodic polarization was achieved in both micelles-free (OPC) and micellescontaining (PoOPC) specimens, i.e. in $\mathrm{NaCl}$ environment the polymer nano-aggregates do not exert any negative effects on concrete electrical resistivity and cathodic current demand respectively.

Microstructural changes (mainly in terms of porosity and pore size distribution) are normally accompanying cathodic protection applications. In the case of CP prevention, these changes would be less pronounced (a thorough investigation, including microstructure at the steel/cement paste interface is on-going).

For both CPre and pCPre conditions, EDX analysis reveals no corrosion products and minimum (or chemically bound only) chlorides in the immediate vicinity of the steel/cement paste interface. 


\section{REFERENCES}

1. T.P. Hoar, Corr. Sci. 7, 341, (1967).

2. M.G. Alvarez, J.R. Galvele, Corr. Sci. 24, 27 (1984).

3. C.L. Page, in "Concrete Solutions II”, St.Malo, France, 194 (2006).

4. J.P. Broomfield, Corrosion of Steel in Concrete, E and FN Spon, London (1997).

5. A.C. Roberts, G.K. Glass, N. Davison, in "Concrete Solutions II", St.Malo, France, 295 (2006).

6. P. Pedeferri, Constr. Build. Mater. 10, 391 (1996).

7. C.L. Page, G. Sergi, ASCE Journal of Materials in Civil Engineering, 12, 8 (2000).

8. G. K. Glass, W. K. Green, J. R. Chadwick, Proceedings of Corrosion 91, Institute of Corrosion, Leighton Buzzard, UK, 3 (1991).

9. BS EN 12696, Cathodic Protection of Steel in Concrete. British Standards Institution, London (2000).

10. G. K. Glass and N. R. Buenfeld, Corr. Sci. 39, 1001 (1997).

11. EU Patent Application - 05077396.1/EP077395, "Pulse Cathodic Protection of reinforced Concrete", EPO (2005).

12. D.A. Koleva, J.H.W de Wit, K.van Breugel, E.van Westing, Z. Lodhi, J. Electrochem. Soc. 154(4), P52 (2007)

13. D.A. Koleva, J.H.W de Wit, G. Ye, K.van Breugel, Z. Lodhi, J. Electrochem. Soc. 154(5), C261 (2007).

14. D. A. Koleva, Z. Guo, K. van Breugel, J. H.W. de Wit, Materials and Corrosion, 60(5), 344 (2009).

15. D.A.Koleva, K.van Breugel, J.M.C. Mol, J.H.W. de Wit, Chloride-induced corrosion in reinforced mortar, modified with core-shell micelles, 216th Meeting of the Electrochemical Society, Vienna, Austria, Session A2 "Nanotechnology General”, paper No 152 (2009).

16. J. Bennett, T. Turk, Report No SHRP-S-359, SHRP, Washington D.C. (1994).

17. M. Pourbaix, Atlas of Electrochemical Equilibrium in aqueous solutions, NACE, 312 (1996).

18. D. Scantlebury, in Proc. 15th Intern. Corrosion Congress, Granada, Spain (2002).

19. H. Davy, Phil. Trans., Royal Society, 114, 151 (1824)

20. D.A. Koleva, K.van Breugel, J.H.W.de Wit, E.van Westing, N.Boshkov, A.Fraaij, J. Electrochem.Soc. 154(3), E45 (2007).

21. D.A. Koleva, O. Copuroglu, K. van Breugel, G. Ye, J.H.W. de Wit, Cem. Concr. Comp., 30, 731 (2008).

22. D.A. Koleva, Z. Guo, K. van Breugel, J.H.W. de Wit, Materials and Corrosion, in press DOI: 10.1002/maco.200905423, (2009).

23. D.A. Koleva, J. Hu, A.L.A. Fraaij, K.van Breugel, J.H.W.de Wit, Cem.Concr.Res., 37 (4), 604 (2007).

24. A.K. Suryavanshi, J..D. Scantlebury, S.B. Lyon, Cem. Concr. Comp. 25, 980 (1995)

25. B. Diaz, X.R. Novoa, M.C. Perez, Cem. Concr. Comp. 28, 237 (2006) 